\title{
A Web-Based Study of the Relationship of Duration of Insulin Pump Infusion Set Use and Fasting Blood Glucose Level in Adults with Type 1 Diabetes
}

\author{
Alysa J. Sampson Perrin, MSPH, Russell C. Guzzetta, BS, Kellee M. Miller, MPH, \\ Nicole C. Foster, MS, Anna Lee, BA, Joyce M. Lee, MD, Jennifer M. Block, RN, CDE, \\ and Roy W. Beck, MD, PhD, for the T1D Exchange Clinic Network
}

\begin{abstract} the infusion set was changed.

\section{Introduction}

$\mathbf{F}$ EW studies have evaluated the impact of duration of insulin pump infusion set use on glycemic control or outlined guidelines for when an infusion set should be changed. ${ }^{1-6}$ A white paper published from the proceedings of an American Association of Diabetes Educators consensus panel noted the lack of literature regarding subcutaneous insulin infusion devices. ${ }^{7}$ To address this gap in evidence, we conducted an Internet-based study using the T1D Exchange's online patient community, Glu (myGlu.org). The study also provided the opportunity to assess the feasibility of using online data collection for longitudinal studies.
\end{abstract}

Background: To evaluate the impact of infusion set use duration on glycemic control, we conducted an Internet-based study using the T1D Exchange's online patient community, Glu (myGlu.org).

Subjects and Methods: For 14 days, 243 electronically consented adults with type 1 diabetes (T1D) entered online that day's fasting blood glucose (FBG) level, the prior day's total daily insulin (TDI) dose, and whether

Results: Mean duration of infusion set use was 3.0 days. Mean FBG level was higher with each successive day of infusion set use, increasing from $126 \mathrm{mg} / \mathrm{dL}$ on Day 1 to $133 \mathrm{mg} / \mathrm{dL}$ on Day 3 to $147 \mathrm{mg} / \mathrm{dL}$ on Day 5 $(P<0.001)$. TDI dose did not vary with increased duration of infusion set use.

Conclusions: Internet-based data collection was used to rapidly conduct the study at low cost. The results indicate that FBG levels increase with each additional day of insulin pump infusion set use.

\section{Subjects and Methods}

Participants were recruited from the T1D Exchange's online patient community, Glu. E-mails were sent to Glu members who indicated a preference to be contacted about research studies. Additionally, information about the study was provided on Glu, Facebook, and Twitter. Eligibility criteria included age $\geq 18$ years, diagnosis of type 1 diabetes (T1D), and daily use of an insulin pump. Participants signed an institutional review board-approved electronic informed consent form. Participants received no compensation for taking part in the study.

Participants completed a questionnaire on their diabetes history, diabetes management, pump use, and most recent hemoglobin A1c (HbA1c) level. For 14 days, participants received daily e-mail prompts to report the following information using a computer or other device: that day's fasting blood glucose (FBG) level, the prior day's total daily insulin (TDI) dose, and whether the infusion set was changed.

To be included in the analyses, a participant must have completed data entry at least 3 of the 14 days. When infusion set use was more than 5 days, only the first 5 days were analyzed owing to the small number of infusion sets used for more than 5 days.

\section{Statistical methods}

Linear regression models were used to examine the association between (1) typical duration of infusion set wear and

\footnotetext{
${ }^{1}$ Jaeb Center for Health Research, Tampa, Florida.

${ }^{2}$ Unitio, Boston, Massachusetts.

${ }^{3}$ University of Michigan, Ann Arbor, Michigan

${ }^{4}$ Stanford University, Stanford, California.
} 
Table 1. Insulin Pump Use Prior to Study

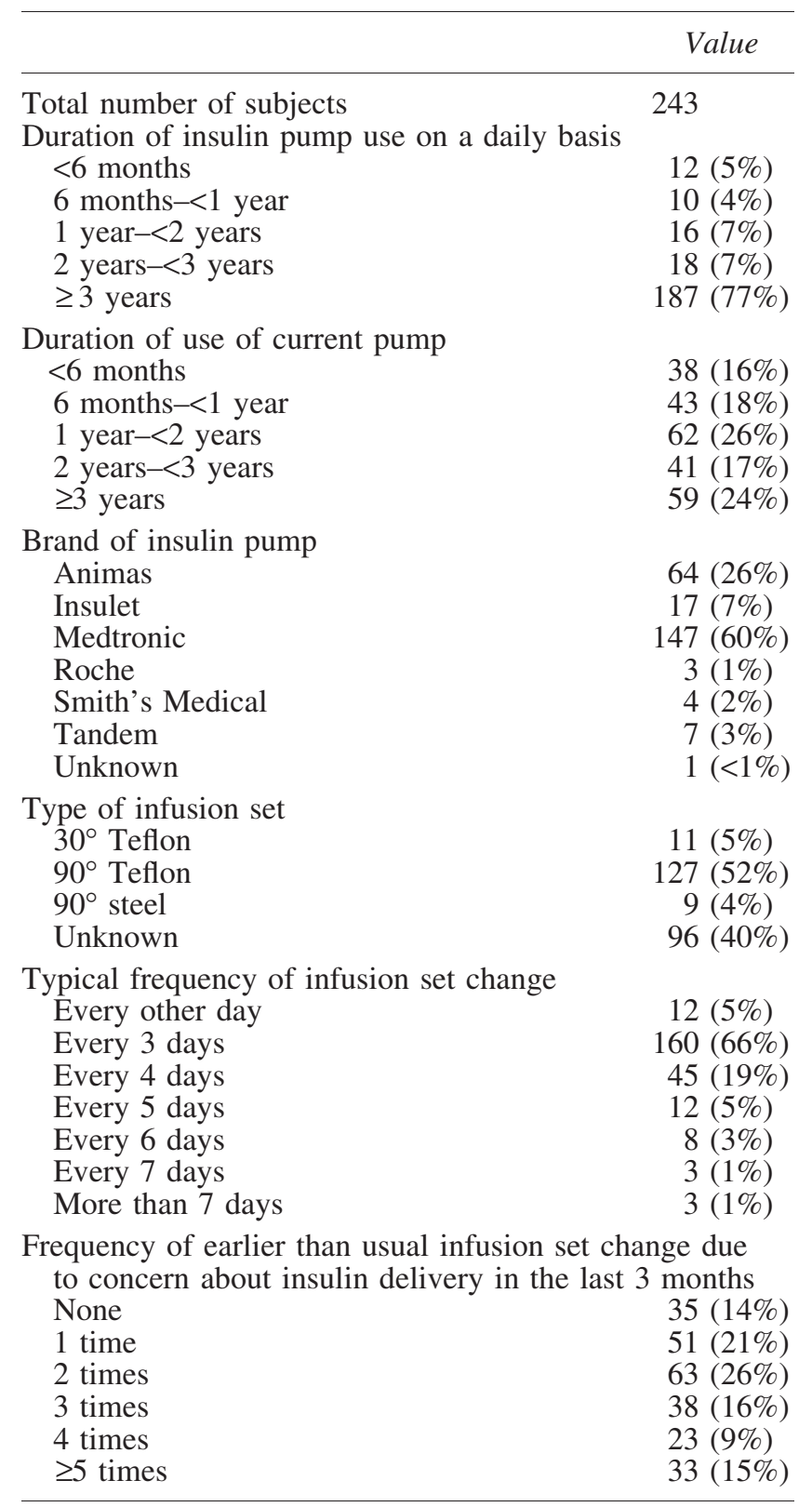

annual household income and (2) self-reported HbA1c and typical duration of infusion set wear. Linear mixed models with repeated measures were used to separately examine the association between FBG and TDI dose, with day of infusion set use accounting for the correlation due to multiple measures per participant. To account for possible confounding due to the potential for participants with higher FBG levels to wear infusion sets longer, average duration of infusion set wear was included as an adjustment covariate in the model. The analyses were repeated in the participant subset that completed all 14 daily questionnaires, with results similar to those from the main analysis (data not shown).

Data analyses used SAS software, version 9.3 (2011; SAS Institute Inc., Cary, NC). All $P$ values are two-sided.

\section{Results}

Of the 317 participants who completed the electronic consent process, $14(4 \%)$ did not complete the enrollment questionnaire, 45 (14\%) failed to enter any daily study data, and $15(5 \%)$ completed $<3$ days of data collection. A cohort of 243 participants was included in the study analyses. Among the 243 participants, $173(71 \%)$ enrolled on the day the study was announced, $29(12 \%)$ on the second day, eight $(3 \%)$ on the third day, and the remaining 33 (14\%) within 11 days.

Mean age of the 243 participants was $42 \pm 14$ years $(13 \%$, 18-25 years; 36\%, 26-40 years; 41\%, 41-60 years; and 11\%, $\geq 61$ years), median duration of diabetes was 22 years (interquartile range, 12-35 years), 91\% were non-Hispanic white, $73 \%$ were female, and $29 \%$ had an annual household income of $\geq \$ 100,000(10 \%,<\$ 35,000 ; 25 \%, \$ 35,000-$ $<\$ 75,000$; and $19 \%, \$ 75,000-<\$ 100,000 ; 16 \%$ did not provide annual income information). Among study participants, $63 \%$ reported using a continuous glucose monitor. Mean HbA1c level was $6.9 \pm 1.1 \%$. Typical duration of infusion set use was not related to income; mean typical duration of infusion set use was $3.7,3.3,3.5$, and 3.5 days for annual income of $<\$ 35,000, \$ 35,000-<\$ 75,000, \$ 75,000-<\$ 100,000$, and $\geq \$ 100,000$, respectively $(P=0.58)$. Mean HbA1c level was $7.0 \%$ for participants typically changing the infusion set every 2-3 days and $6.7 \%$ for those who changed every 4 or more days $(P=0.03)$.

The types of insulin pumps used by the participants are shown in Table 1. Insulin pump use was at least 3 years

Data are number $(\%)$.

Table 2. Glycemic Control and Insulin Amount According to Day of Infusion Set Use

\begin{tabular}{|c|c|c|c|c|c|}
\hline & Day 1 & Day 2 & Day 3 & Day 4 & Day 5 \\
\hline \multicolumn{6}{|l|}{ Fasting blood glucose (mg/dL) } \\
\hline$n$ & 236 & 236 & 230 & 163 & 77 \\
\hline Mean \pm SD & $125 \pm 39$ & $128 \pm 44$ & $133 \pm 49$ & $140 \pm 56$ & $147 \pm 68$ \\
\hline Median (interquartile range) & $119(100,146)$ & $116(99,149)$ & $125(97,158)$ & $132(94,171)$ & $128(99,174)$ \\
\hline$\%>180 \mathrm{mg} / \mathrm{dL}$ & $9 \%$ & $13 \%$ & $15 \%$ & $21 \%$ & $22 \%$ \\
\hline$\%>250 \mathrm{mg} / \mathrm{dL}$ & $1 \%$ & $1 \%$ & $3 \%$ & $4 \%$ & $8 \%$ \\
\hline \multicolumn{6}{|l|}{ Total daily insulin (units) } \\
\hline$n$ & 236 & 230 & 163 & 77 & 40 \\
\hline Mean \pm SD & $40.1 \pm 20.5$ & $39.6 \pm 19.5$ & $37.9 \pm 16.6$ & $36.7 \pm 16.4$ & $39.3 \pm 15.8$ \\
\hline Median (interquartile range) & $35.6(27.0,45.4)$ & $34.9(27.0,45.5)$ & $34.5(28.0,45.5)$ & $35.0(27.0,43.0)$ & $37.5(29.5,46.5)$ \\
\hline
\end{tabular}

When infusion set use was more than 5 days, only the first 5 days were analyzed because of the small number of infusion sets used more than 5 days. 
Table 3. Fasting Blood Glucose by Day of Infusion Set Wear for Subsets of Duration of Infusion Set Wear

Fasting blood glucose level ( $\mathrm{mg} / \mathrm{dL})$

\begin{tabular}{|c|c|c|c|c|c|}
\hline \multirow{2}{*}{ Days of infusion set wear } & & & & & \\
\hline & Day 1 & Day 2 & Day 3 & Day 4 & Day 5 \\
\hline \multicolumn{6}{|l|}{$3(n=67)$} \\
\hline Mean \pm SD & $125 \pm 33$ & $133 \pm 44$ & $142 \pm 53$ & - & - \\
\hline Median (interquartile range) & $122(104,145)$ & $121(104,152)$ & $131(101,172)$ & - & - \\
\hline \multicolumn{6}{|l|}{$4(n=86)$} \\
\hline Mean \pm SD & $127 \pm 42$ & $127 \pm 42$ & $132 \pm 44$ & $148 \pm 60$ & - \\
\hline Median (interquartile range) & $116(100,142)$ & $115(100,148)$ & $123(95,161)$ & $142(99,180)$ & - \\
\hline \multicolumn{6}{|l|}{$5(n=75)$} \\
\hline Mean \pm SD & $125 \pm 41$ & $125 \pm 47$ & $125 \pm 49$ & $131 \pm 54$ & $147 \pm 68$ \\
\hline Median (interquartile range) & $120(92,151)$ & $113(91,149)$ & $118(91,142)$ & $122(90,155)$ & $128(98,174)$ \\
\hline
\end{tabular}

When infusion set use was more than 5 days, only the first 5 days were analyzed because of the small number of infusion sets used more than 5 days.

Table 4. Glycemic Control by Demographic and Clinical Factors According to Day of Infusion Set Use

\begin{tabular}{|c|c|c|c|c|c|c|c|c|c|c|c|c|}
\hline \multirow[b]{3}{*}{ Factor } & \multicolumn{12}{|c|}{ Fasting blood glucose (mg/dL) } \\
\hline & \multicolumn{2}{|r|}{ Day 1} & \multicolumn{2}{|r|}{ Day 2} & \multicolumn{2}{|r|}{ Day 3} & \multicolumn{2}{|r|}{ Day 4} & \multicolumn{2}{|r|}{ Day 5} & \multicolumn{2}{|c|}{$\mathrm{P}$ value } \\
\hline & $\mathrm{n}$ & Mean $\pm S D$ & $\mathrm{n}$ & Mean $\pm S D$ & $\mathrm{n}$ & Mean $\pm S D$ & $\mathrm{n}$ & $M e a n \pm S D$ & $\mathrm{n}$ & $M e a n \pm S D$ & Unadjusted $^{\mathrm{a}}$ & Adjusted $^{\mathrm{b}}$ \\
\hline Gender & & & & & & & & & & & 0.30 & 0.64 \\
\hline Female & 173 & $127 \pm 39$ & 173 & $129 \pm 45$ & 170 & $135 \pm 52$ & 122 & $138 \pm 56$ & 65 & $148 \pm 69$ & & \\
\hline Male & 63 & $122 \pm 39$ & 63 & $124 \pm 43$ & 60 & $128 \pm 41$ & 41 & $146 \pm 63$ & 12 & $141 \pm 61$ & & \\
\hline Age (years) & & & & & & & & & & & 0.07 & 0.63 \\
\hline $18-25$ & 30 & $146 \pm 40$ & 30 & $139 \pm 50$ & 29 & $136 \pm 51$ & 21 & $140 \pm 52$ & 11 & $133 \pm 44$ & & \\
\hline $26-40$ & 83 & $123 \pm 37$ & 83 & $128 \pm 43$ & 81 & $138 \pm 52$ & 62 & $144 \pm 59$ & 36 & $138 \pm 58$ & & \\
\hline $41-60$ & 97 & $124 \pm 40$ & 97 & $123 \pm 44$ & 95 & $130 \pm 49$ & 66 & $136 \pm 61$ & 28 & $168 \pm 84$ & & \\
\hline$\geq 61$ & 26 & $114 \pm 37$ & 26 & $131 \pm 41$ & 25 & $128 \pm 35$ & 14 & $137 \pm 52$ & 2 & $105 \pm 30$ & & \\
\hline Race/ethnicity & & & & & & & & & & & 0.33 & 0.50 \\
\hline White non-Hispanic & 216 & $126 \pm 40$ & 216 & $127 \pm 45$ & 212 & $133 \pm 49$ & 150 & $139 \pm 57$ & 68 & $151 \pm 69$ & & \\
\hline Black non-Hispanic & 1 & 92 & 1 & 130 & 1 & 70 & 1 & 138 & 1 & 65 & & \\
\hline Hispanic or Latino & 8 & $102 \pm 29$ & 8 & $121 \pm 29$ & 7 & $116 \pm 36$ & 5 & $178 \pm 101$ & 3 & $130 \pm 77$ & & \\
\hline Other race/ethnicity & 11 & $131 \pm 35$ & 11 & $145 \pm 43$ & 10 & $150 \pm 57$ & 7 & $124 \pm 33$ & 5 & $126 \pm 45$ & & \\
\hline $\begin{array}{l}\text { Type } 1 \text { diabetes } \\
\text { duration (years) }\end{array}$ & & & & & & & & & & & 0.03 & 0.22 \\
\hline$\leq 9$ & 46 & $131 \pm 48$ & 46 & $125 \pm 43$ & 45 & $130 \pm 47$ & 37 & $130 \pm 54$ & 17 & $129 \pm 39$ & & \\
\hline $10-\leq 20$ & 57 & $137 \pm 37$ & 57 & $141 \pm 48$ & 55 & $139 \pm 52$ & 36 & $160 \pm 74$ & 15 & $125 \pm 64$ & & \\
\hline $21-\leq 31$ & 63 & $129 \pm 38$ & 63 & $127 \pm 45$ & 62 & $143 \pm 55$ & 46 & $149 \pm 46$ & 26 & $148 \pm 53$ & & \\
\hline$>31$ & 70 & $110 \pm 31$ & 70 & $119 \pm 40$ & 68 & $122 \pm 39$ & 44 & $122 \pm 51$ & 19 & $181 \pm 96$ & & \\
\hline Annual income & & & & & & & & & & & 0.74 & 0.65 \\
\hline$<\$ 35,000$ & 22 & $136 \pm 52$ & 22 & $145 \pm 50$ & 22 & $129 \pm 57$ & 16 & $145 \pm 87$ & 10 & $147 \pm 63$ & & \\
\hline$\$ 35,000-<\$ 75,000$ & 63 & $125 \pm 36$ & 63 & $123 \pm 40$ & 62 & $125 \pm 45$ & 43 & $132 \pm 45$ & 17 & $154 \pm 77$ & & \\
\hline$\$ 75,000-<\$ 100,000$ & 45 & $121 \pm 37$ & 45 & $125 \pm 48$ & 44 & $137 \pm 50$ & 32 & $141 \pm 53$ & 18 & $154 \pm 70$ & & \\
\hline$\geq \$ 100,000$ & 68 & $124 \pm 34$ & 68 & $126 \pm 43$ & 66 & $133 \pm 46$ & 43 & $144 \pm 48$ & 17 & $139 \pm 59$ & & \\
\hline Not provided & 38 & $128 \pm 48$ & 38 & $132 \pm 45$ & 36 & $145 \pm 54$ & 29 & $141 \pm 74$ & 15 & $141 \pm 75$ & & \\
\hline Brand of insulin pump & & & & & & & & & & & 0.43 & 0.88 \\
\hline Animas & 62 & $133 \pm 42$ & 62 & $130 \pm 39$ & 61 & $132 \pm 47$ & 44 & $138 \pm 56$ & 20 & $139 \pm 52$ & & \\
\hline Insulet & 17 & $121 \pm 34$ & 17 & $118 \pm 37$ & 16 & $125 \pm 52$ & 5 & $139 \pm 54$ & 0 & - & & \\
\hline Medtronic & 143 & $124 \pm 39$ & 143 & $129 \pm 48$ & 139 & $135 \pm 50$ & 102 & $139 \pm 59$ & 53 & $154 \pm 74$ & & \\
\hline Roche & 2 & $126 \pm 17$ & 2 & $111 \pm 51$ & 2 & $127 \pm 47$ & 2 & $212 \pm 28$ & 1 & 123 & & \\
\hline Smith's Medical & 4 & $96 \pm 15$ & 4 & $106 \pm 23$ & 4 & $98 \pm 30$ & 4 & $126 \pm 34$ & 2 & $96 \pm 43$ & & \\
\hline Tandem & 7 & $126 \pm 26$ & 7 & $121 \pm 50$ & 7 & $151 \pm 58$ & 5 & $159 \pm 66$ & 0 & - & & \\
\hline Type of insulin ${ }^{c}$ & & & & & & & & & & & 0.87 & 0.71 \\
\hline Apidra (insulin glulisine) & 22 & $122 \pm 35$ & 22 & $128 \pm 50$ & 20 & $158 \pm 73$ & 11 & $143 \pm 55$ & 2 & $153 \pm 91$ & & \\
\hline Humalog (insulin lispro) & 109 & $126 \pm 38$ & 109 & $133 \pm 44$ & 108 & $136 \pm 48$ & 76 & $139 \pm 50$ & 33 & $152 \pm 65$ & & \\
\hline Novolog (insulin aspart) & 104 & $126 \pm 42$ & 104 & $123 \pm 43$ & 101 & $125 \pm 43$ & 75 & $141 \pm 66$ & 41 & $145 \pm 71$ & & \\
\hline
\end{tabular}

${ }^{a}$ Unadjusted $P$ values from a linear mixed model with repeated measures adjusted for average duration of infusion set wear. Only the adjustment factor, day of infusion set wear, and the indicated factor were included in the model. Age and duration of type 1 diabetes were included as continuous factors.

${ }^{\mathrm{b}}$ Adjusted $P$ values from a linear mixed model with repeated measures adjusted for average duration of infusion set wear. In addition to the adjustment factor and day of infusion set wear, all factors in the table were included in model. Age and duration of type 1 diabetes were included as continuous factors.

${ }^{\mathrm{c}}$ One participant reported using Humalog R U-500. 
in $77 \%$ of participants, with $66 \%$ of participants usually changing their infusion set every 3 days.

\section{Outcome data}

Daily data were entered for all 14 days by $213(71 \%)$ of the 243 participants, 10-13 days by eight (3\%), 6-9 days by 12 (5\%), and 3-5 days by 10 (4\%). During the study, the 243 participants reported using a total of 688 infusion sets. Mean duration of infusion set use during the study was $3.0 \pm 1.0$ days: $1-2$ days for $26 \%, 3$ days for $48 \%, 4$ days for $19 \%$, and 5 days for $7 \%$.

Mean FBG increased with each additional day of infusion set wear, increasing from $125 \mathrm{mg} / \mathrm{dL}$ on Day 1 to $133 \mathrm{mg} / \mathrm{dL}$ on Day 3 to $147 \mathrm{mg} / \mathrm{dL}$ on Day $5(P<0.001)$ (Table 2$)$. It is interesting that for infusion sets used for 4 days, there was not a substantial increase in the mean FBG until Day 4, and for infusion sets used for 5 days, there was not a substantial increase until Day 5 (Table 3). Other than duration of infusion set use, no other factors were associated with the increase in mean glucose level as seen from Day 1 to Day 5 (Table 4). Mean TDI dose remained relatively constant from Day 1 to Day 5 (from 40 to 39 units/day) $(P=0.94)$.

\section{Discussion}

Our study used online data collection to address an important clinical question much more rapidly and at lower cost than comparable clinic-based studies. Within 2 days of announcing the study, more than 200 participants had enrolled, and the study was essentially completed within 1 month of initiation. Participants were not compensated, so costs for the study were limited to programming for the Web site's data collection capabilities and data analyses. The study's main finding was that FBG levels increased with each day of infusion set use. It is particularly noteworthy that for those infusion sets used for 4 and 5 days, mean FBG only modestly increased until Day 4 and 5 , respectively. Thus patients seem to be able to autotitrate the frequency in which they need to change their infusion sites.

Our findings are consistent with a small prior study conducted in 20 adults with T1D that found that from Day 2 to Day 5 of infusion set use, FBG increased from 120 to $155 \mathrm{mg} /$ dL. ${ }^{8}$ In that study, TDI dose increased from $48.5 \pm 11.8$ units to $55.3 \pm 17.9$ units, whereas we did not find an increase in TDI dose in our subjects.

In assessing the feasibility of conducting a study using online data collection, potential biases that may be present depending on the characteristics of the participants and the lack of direct monitoring by clinic personnel may require further evaluation. In the case of the current study, even though our participants were better controlled than most adults with T1D in the United States and more likely to be using continuous glucose monitoring, ${ }^{9}$ bias with respect to the study participants seems unlikely to have had a major effect on the results because no other factors were found to be associated with the increase in FBG over time. Additionally, because data were entered daily by participants, it seems unlikely that study participants would have falsely entered FBG values. Although FBG data were not verified, a study such as this could have done so if it was required through the uploading of blood glucose meter data at the end of the study.

In conclusion, this study demonstrates the power of using online data collection and a T1D social network to conduct longitudinal clinical research studies rapidly at low cost. The results of the study indicate that FBG levels increase each day of successive insulin pump infusion set use, which is important for insulin pump users and their healthcare providers to recognize.

\section{Acknowledgments}

This work was supported through the Leona M. and Harry B. Helmsley Charitable Trust.

\section{Author Disclosure Statement}

R.W.B.'s nonprofit employer has received consultant payments on his behalf from Sanofi and Animas and a research grant from NovoNordisk with no personal compensation to him. A.J.S.P., R.C.G., K.M.M., N.C.F., A.L., J.M.L., and J.M.B. declare no competing financial interests exist.

A.J.S.P., R.C.G., A.L., J.M.L., J.M.B., and R.W.B. researched data, wrote, and reviewed/edited the manuscript. K.M.M. and N.C.F. performed statistical analyses, wrote, and reviewed/edited the manuscript.

\section{References}

1. Frid A, Hirsch L, Gaspar R, et al.: New injection recommendations for patients with diabetes. Diabetes Metab 2010; 36(Suppl 2):S3-18.

2. Frid A, Hirsch L, Gaspar R, et al.: The Third Injection Technique Workshop in Athens (TITAN). Diabetes Metab 2010;36(Suppl 2):S19-S29.

3. Heinemann L, Krinelke L: Insulin infusion set: the Achilles heel of continuous subcutaneous insulin infusion. J Diabetes Sci Technol 2012;6:954-964.

4. Phillip M, Battelino T, Rodriguez $\mathrm{H}$, et al.: Use of insulin pump therapy in the pediatric age-group: consensus statement from the European Society for Paediatric Endocrinology, the Lawson Wilkins Pediatric Endocrine Society, and the International Society for Pediatric and Adolescent Diabetes, endorsed by the American Diabetes Association and the European Association for the Study of Diabetes. Diabetes Care 2007;30:1653-1662.

5. Scheiner G, Sobel RJ, Smith DE, et al.: Insulin pump therapy: guidelines for successful outcomes. Diabetes Educ 2009;35(Suppl 2):29S-41S; quiz 28S, 42S-43S.

6. Schmid V, Hohberg C, Borchert M, et al.: Pilot study for assessment of optimal frequency for changing catheters in insulin pump therapy - trouble starts on day 3. J Diabetes Sci Technol 2010;4:976-982.

7. Scheiner G, Sage D, Wolff P, et al.: Insulin Pump Therapy: Best Practices in Choosing and Using Infusion Devices. Chicago: American Association of Diabetes Educators, 2011.

8. Thethi TK, Rao A, Kawji H, et al.: Consequences of delayed pump infusion line change in patients with type 1 diabetes mellitus treated with continuous subcutaneous insulin infusion. J Diabetes Complications 2010;24:73-78.

9. Beck RW, Tamborlane WV, Bergenstal RM, et al.: The T1D Exchange Clinic Registry. J Clin Endocrinol Metab 2012; 97:4383-4389.

Address correspondence to: Alysa J. Sampson Perrin, MSPH Jaeb Center for Health Research 15310 Amberly Drive, Suite 350

Tampa, FL 33647

E-mail: aperrin@jaeb.org 


\section{This article has been cited by:}

1. Anneke Graf, Sybil A. McAuley, Catriona Sims, Johanna Ulloa, Alicia J. Jenkins, Gayane Voskanyan, David N. O’Neal. 2017. Moving Toward a Unified Platform for Insulin Delivery and Sensing of Inputs Relevant to an Artificial Pancreas. Journal of Diabetes Science and Technology 11:2, 308-314. [Crossref]

2. Karlin Andrew W., Ly Trang T., Pyle Laura, Forlenza Gregory P., Messer Laurel, Wadwa R. Paul, DeSalvo Daniel J., Payne Sydney L., Hanes Sarah, Clinton Paula, Maahs David M., Buckingham Bruce. 2016. Duration of Infusion Set Survival in Lipohypertrophy Versus Nonlipohypertrophied Tissue in Patients with Type 1 Diabetes. Diabetes Technology \& Therapeutics 18:7, 429-435. [Abstract] [Full Text HTML] [Full Text PDF] [Full Text PDF with Links] [Supplemental Material]

3. Heinemann Lutz. 2016. Insulin Infusion Sets: A Critical Reappraisal. Diabetes Technology \& Therapeutics 18:5, 327-333. [Abstract] [Full Text HTML] [Full Text PDF] [Full Text PDF with Links]

4. Eda Cengiz, Bruce Bode, Michelle Van Name, William V Tamborlane. 2016. Moving toward the ideal insulin for insulin pumps. Expert Review of Medical Devices 13:1, 57-69. [Crossref] 